\title{
,Fact and Fiction' in der mittelalterlichen arabischen Literatur. Anmerkungen zu einer Debatte
}

Isabel Toral-Niehoff

\begin{abstract}
Wer ist wohl frevelhafter als einer, der sich anschickt, so etwas wie meine Schöpfung zu schaffen! Sollen sie doch einmal ein Körnchen schaffen, sollen sie doch eine kleine Ameise schaffen! ${ }^{1}$
\end{abstract}

Die These von Walter Haug über die,Geburt der Fiktionalität' im Mittelalter, nach welcher diese erst mit dem höfischen Roman Chrétien de Troyes erfunden worden sei, hat eine umfangreiche Diskussion in der europäischen Mediävistik ausgelöst. Diese befasst sich mit der Frage, ab wann, ob und wie von Fiktionalität, Fingieren und Fiktion in der europäischen Literatur zu reden sei, mit der Folge, dass diese Begriffe eine zunehmende Historisierung und Kulturalisierung erfahren haben (Haug 2003; Müller 2004: 281-283 und passim).

Dieselbe Frage lässt sich aber auch für nichteuropäische, vormoderne Literaturen stellen, wie zum Beispiel für die umfangreiche arabischsprachige Literatur des Mittelalters (8.-12. Jhd.). ${ }^{2}$ Gibt es Fiktionalität in der mittelalterlichen hochsprachlichen arabischen Literatur (im Folgenden: MHAL), die sich einer Faktualität gegenüberstellen ließe? ${ }^{3}$ Lässt sich hier von einer Opposition zwischen fiktionalem und faktualem Erzählen (Genette 1990) sprechen? Wird hier vielleicht ein der europäischen Moderne entnommener Begriff übertragen?

1 Zitiert nach Heinrichs (1969: 39). Es handelt sich um einen hadīth qudsī, somit nach islamischer Auffassung um eine außerkoranische göttliche Offenbarung, die dem Propheten durch Inspiration zuteil wurde, ihr kommt deshalb ein besonderer Stellenwert zu. Erkennbar ist, dass hier kein Unterschied zwischen menschlicher und göttlicher Schöpfung gemacht wird und somit auch theologische Gründe für ein ,Verbot' von Fiktion angeführt werden konnten.

2 Diese Zeit entspricht der ersten Blütezeit der arabisch-islamischen Kultur in der späten Umayyaden- und frühen Abbasidenzeit (auch als ,Goldenes Zeitalter' bekannt). Es gibt eine umfangreiche (hier nicht relevante) Diskussion in der Fachwelt darüber, ob die Bezeichnung ,mittelalterlich' im arabischen Kontext sinnvoll ist - im Rahmen dieses interdisziplinären Bandes erschien sie mir jedoch geeignet, um diese Texte chronologisch korrekt zuzuordnen. Alternative Bezeichnungen sind ,klassische Arabische Literatur' und ,canonical'. Ich beziehe mich im Folgenden nur auf die hochsprachliche arabische Literatur.

3 Diese Dichotomie wird neuerdings auch für das europäische Mittelalter in Frage gestellt (Müller 2004: 282). 


\section{Fiktion und Fiktionalität in der mittelalterlichen arabischen Literatur - Probleme}

Die Diskussion über Fiktion und Fiktionalität in der MHAL hat nicht einmal ansatzweise die Komplexität und Differenziertheit wie in der Mediävistik erreicht. ${ }^{4}$ Dies liegt auch daran, dass sich die Fachwelt allgemein darin einig ist, dass in der hochsprachlichen Literatur Fiktion abgelehnt wird bzw. kaum existiert. ${ }^{5}$ Sie fände sich zwar häufig in volkstümlichen Epen und Märchen, diese Genres werden aber traditionell in der arabischen Literatur als wertlose Trivialliteratur betrachtet, auch wenn sie sehr populär waren. ${ }^{6}$

Schauen wir in eines der einschlägigen Handbücher des Faches, in die Encyclopaedia of Arabic Literature, lesen wir z. B. unter dem Lemma „fiction, medieval“ gleich zu Beginn Folgendes: „Canonical Arabic literature is basically rejective of fiction“ (Meisami 1998: 228b). Diese Einschätzung stützt sich einerseits auf kritische Aussagen arabischer Gelehrten zum Thema ,Erfinden' und ,Lügen' in der Literatur; ${ }^{7}$ andererseits auf die Tatsache, dass die ansonsten hoch entwickelte autochthone arabische Literaturtheorie bis auf wenige Ausnahmen keine Reflexion über ,Fiktion' bzw. eventuelle äquivalente Konzepte zu kennen scheint. ${ }^{8}$ Es sind auch nur wenige hochsprachliche narrative Texte dieser Epoche erhalten, die in eine literarische Welt der Fiktion verweisen, also eine ,autonome Fiktionalität ${ }^{\star}$ aufweisen, in der die Fiktion auch explizit gemacht bzw. ,entblößt ${ }^{6}$ wird. ${ }^{9}$ Fiktion ist nach allgemeiner Einschätzung der Forschung in der MHAL der Dichtung vorbehalten („Die beste Dichtung ist diejenige, die am meisten lügt");10 während

4 Für einen Überblick über die Positionen in der Mediävistik s. Müller (2004).

5 S. die Auflistung der Stellungnahmen bei Leder (1998: 34 f.), dort vor allem unter Bezugnahme auf Heinrichs (1969: 32-55) und van Grunebaum (1947). Vgl. hierzu allgemein auch Hoyland (2006), Drory (1994), Bonebakker (1992).

6 Vgl. Heath (1990) zu den Besonderheiten und Schwierigkeiten der Erforschung dieser Literatur.

7 Der locus classicus sind die abwertenden Aussagen von dem Bagdader Bibliographen Ibn alNadīm (gest. ca. 998) zu den Hazar Afshān (die Frühform der 1001 Nacht-Erzählungen) in seinem Kitāb al-Fibrist (Ibn al-Nadìm 1871/2: 304); vgl. auch das ähnlich abwertende Zitat von al-Nahrawānī bei Drory (1994: 156).

8 Eine wichtige Ausnahme bildet der von Wolfhart Heinrichs 1969 detailliert untersuchte Literaturkritiker al-Qartağannī (1211-1285), der eine sehr differenzierte poetische Lehre zur Frage von Inspiration, Einbildungskraft und ,Erfindung' in der Literatur entwickelt hat (ikbtirā $\overline{\text { ) }}$, die sich allerdings vor allem auf Fiktion in der Poesie bezieht (Heinrichs 1969, passim). Zur Fiktion in der Poesie s. auch den Überblick bei Kennedy (2005a).

9 Ausnahmen bilden erstens das Genre der Maqāmen (Meisami 1998: 506a-508b), eine Art Picaro-Romane in Reimprosa, entwickelt im 11. Jahrhundert, die explizit fiktive Personen enthielten; zweitens sind auch Tierfabeln überliefert (z. B. Kalīla wa Dimna). In beiden Fällen scheinen sie ihre Akzeptanz der Tatsache zu verdanken, dass hier keine Illusion von Wirklichkeit suggeriert wurde, so dass auch keine Irritation entstehen konnte (Meisami 1998: 228b). Zum Begriff ,autonome Fiktionalität‘s. Müller (2004: 282-83).

10 „Ahsanu al-shicr akdhabubu“ - Ein oft zitierter Spruch, hier zitiert nach Meisami (1998: 228b). Mit ,Lüge' ist hier wohl literarische Fiktion i. S. von rhetorischer Stilisierung durch 
sie wie erwähnt in Erzähltexten in die abgewertete Welt der trivialen Unterhaltungs- und Volksliteratur verbannt wird. ${ }^{11}$ Die Umstände und Gründe dieser Ablehnung sind noch nicht erschöpfend untersucht; sie scheint auch nicht von Anbeginn wirksam gewesen zu sein und sich auf die vormoderne Literatur in arabischer Sprache zu beschränken. So war die neupersische Literatur nicht davon betroffen - man denke z. B. an angesehene Autoren wie Nezāmī (1141-1205?), der umfangreiche Liebesepen schrieb, die in den Kanon ,klassischer Texte ${ }^{6}$ aufstiegen (Bürgel 1990: 306-310). Die umfangreiche Liste von fiktionalen Erzähltexten in dem Buchverzeichnis des Bibliographen Ibn al-Nadìm (gest. ca. 998) zeigt auch, dass sehr viele dieser als ,trivial' betrachteten Werke noch Ende des 10ten Jahrhunderts in Bagdad zirkulierten. Allerdings sind fast keine davon erhalten, vermutlich weil sie irgendwann nicht mehr als überlieferungswert betrachtet wurden. ${ }^{12}$ Das Verschwinden dieser Schriften schreibt Wolfhart Heinrichs der so genannten sunnitischen ,Reaktion' in der Seldschukenzeit des 11. und 12. Jahrhunderts zu, erklärt sie also mit ideologischen und sozialen Verschiebungen, infolge derer eine andere Bildungsschicht den Geltungsanspruch dieser Texte bestritt. ${ }^{13}$

Der narrative Stil der frühen Prosa-Texte präsentiert sich zudem realistischfaktual, ${ }^{14}$ was den Eindruck verstärkt, Fiktion habe es in der MHAL nicht gegeben. Diese Texte folgen dabei der sogenannten khabar-Form, die hier kurz beschrieben sei: Sie sind molekular strukturiert, d. h. sie sind in einzelne Nachrichten oder $k b a-$ bar (Plural $a k b b \bar{a} r$ ) gegliedert. Eine khabar besteht aus einer bis zwei narrativ in sich geschlossenen Episoden, das Erzähltempo ist rasch, die beschreibenden Passagen sind kurz, es überwiegen Dialoge, die auch das innere Erleben der Personen illustrieren (szenische Erzählsituation bzw. ,showing'), die Fokalisierung ist extern; der Erzähler gibt eine neutrale Sicht auf die Geschichte und interveniert zumeist auch nicht persönlich in die Handlung, es sei denn, der Erzähler fungiert als Augenzeu-

Metaphern, Hyperbolen usw. gemeint. Zur Poesie als Ort der Fiktion s. auch Kennedy (2005a).

11 Allgemein zur volkstümlichen Literatur Heath (1990). Die volkstümlichen fiktionalen Erzähltexte des Mittelalters sind meist unter dem Namen kburāfät (Meisami 1998: 443b444a) bekannt, angeblich benannt nach einem Lügner namens Khurāfa; hierzu zählten allerlei phantastische Geschichten, die zur Unterhaltung von Kindern, Frauen und sozial Deklassierten dienen sollten. S. auch Leder (1998a: 39-43) „Fantastic stories as ,trivial“ literature"; Hoyland (2006: 17).

12 Diese Liste ist aufgearbeitet in Ghazi (1957).

13 Heinrichs (1969: 40-43). Er verbindet diese fiktionale Literatur mit der iranisierten Schicht der kuttāb bzw. Staatssekretäre; mit dem Aufkommen einer anderen Verwaltungsschicht in der Seldschukenzeit habe man sich auf den, wahren' Islam besinnen wollen, dem diese Elemente angeblich fremd waren.

14 Ich verwende hier dieses Begriffspaar um den Stil der frühen arabischen Prosa zu beschreiben, der realistisch ist, insofern er die Illusion von Wirklichkeitsnähe evozieren möchte (zum Realismus in der Erzählung s. Fludernik 2008: 66-67). Mit dem europäischen Realismus im Roman des 19. Jhds. hat dies allerdings nichts zu tun. Inwiefern durch diesen Stil in der arabischen Prosa auch ein Anspruch auf Faktualität gestellt wurde, ist Thema der hier skizzierten Debatte und soll offen bleiben; mit der Doppelung möchte ich hier genau auf diese Ambivalenz hinweisen. 
ge. ${ }^{15}$ Oft sind diese akbbār von einer Überliefererkette (isnād) begleitet, die als Authentifizierung dient und möglichst lückenlos bis zum ersten Tradenten führt, der idealiter ein Augenzeuge oder zumindest ein Zeitgenosse des Ereignisses ist. ${ }^{16}$ Diese $a k b b \bar{a} r$ werden unabhängig tradiert und erscheinen in modifizierter Form in zahlreichen Kontexten mit unterschiedlichen Funktionen.

Die khabar-Form findet sich in einer weiten Bandbreite von Prosatexten, die zum Teil historiographisch, z. T. geographisch, z. T. eher derjenigen vielgestaltigen Textgruppe zuzuordnen ist, die in der Fachwelt unter der Bezeichnung adab bekannt ist. Es würde hier zu weit führen, die zahlreichen Versuche, diesen schillernden Begriff $a d a b$ zu fassen; am ehesten entspricht diese Gruppe der mittelalterlichen und neuzeitlichen ,Buntschriftstellerei ${ }^{6}{ }^{17}$ Es ist hier wichtig, zu betonen, dass Gattungsgrenzen in der Frühzeit der arabischen Prosa nur schwer zu ziehen sind, dies gilt auch für die Grenze zwischen Sachtexten, Historiographie und literarischer Prosa. ${ }^{18}$

Diese realistisch-faktuale Darstellung in der khabar-Form steht allerdings oftmals in irritierendem Widerspruch zu dem offenbar fiktiven Inhalt, wie Stefan Leder anmerkt:

The existence of fictive elements $[\ldots]$ cannot seriously be rejected $[\ldots]$ fictional narration, wherever it appears, is embedded in a mainstream of factual, or allegedly factual, narration $[\ldots]$ this ambiguity often obstructs any attempt to decide which text, or which part of a text should be regarded as fiction. (Leder 1998a: 34) ${ }^{19}$

Wie Julie S. Meisami in ihrer Kritik an Stefan Leder allerdings argumentiert hat, könnte diese Diskrepanz auch auf unsere eurozentrische Perspektive zurückgehen, die in ihren kulturell bedingten Vorstellungen von Faktualität und Plausibilität gefangen ist.

This gives the game away: the „European tradition' is the standard against which Arabic prose writing is to be judged. If the Arabs failed to recognize, or to accept „fiction“, we, however, can recognize fiction when we see it. (Meisami 2005: 151)

Diese Irritation muss hier allerdings nicht notwendigerweise auf kulturspezifische Sichtweisen zurückgehen, sondern resultiert vielleicht auch aus unserem modernen Blickwinkel. Im Kern handelt es sich hier nämlich um dieselbe Problematik,

15 Zum ersten Mal genauer beschrieben in Caskel (1930: 34-40); ferner Leder (1992, passim); Leder/Kilpatrick (1992: 10-14); Leder (1998a: 37-38). In der unten besprochenen Episode spricht als Autorität ein Augenzeuge, dennoch interveniert er kaum in die Handlung.

16 U. a. Rosenthal (1968: 66-71), und Robinson (2004), index, s.v. isnād (chains of transmission), s. auch dort (92-97).

17 Hier sei auf Bestimmungsversuche von adab bei Fähndrich (1990) und Bonebakker (1990) verwiesen. Für den Begriff ,Buntschriftstellerei` vgl. neuerdings den Sammelband von Schock (2012).

18 Ähnlich in der europäischen mittelalterlichen Literatur, wo der Übergang zwischen historiographisch, historiographisch fingierendem und fiktionalem Erzählen fließend ist (Müller 2004: 295).

19 Vgl. auch Leder: „non-factual matters are sometimes presented in a manner that discloses their fictional character" (2005: 125). 
die sich auch in der Mediävistik stellt, wenn sie das ,eigentümliche Realitäts- und Wahrheitsverständnis' im Mittelalter diskutiert. Jan Müller gibt in diesem $\mathrm{Zu}-$ sammenhang zu bedenken, dass ,was vom Standpunkt einer anderen Kultur als unwahr erscheint $[\ldots]$ in seinem genuinen Geltungsbereich weder bloße Erfindung noch Lüge“ sei (Müller 2004: 284). Ein ahistorisches Übertragen von modernen europäischen Wahrheitsbegriffen auf die arabische Kultur des Mittelalters sollte also vermieden werden.

Abgesehen von dieser wichtigen Präzisierung ist anzumerken, dass ein realistisch-faktualer Stil kein Hinweis auf einen nicht-fiktionalen Text sein muss (Leder 1998a: 36-39 „Factuality as textual environment“). So hat Daniel Beaumont den khabar-Stil sehr treffend mit dem ,Hard-Boiled'-Stil der amerikanischen Erzählliteratur verglichen (Beaumont 1996), und so verdeutlicht, dass diese vermeintliche ,Faktualität' auch nur eine weitere fiktionale Strategie sein kann. ${ }^{20}$

Weitere Probleme erwachsen aus der Tatsache, dass die Begriffe aus dem Wortfeld ,Fiktion' (z. B. auch ,fiktional', ,fiktiv', ,fiktionalisiert', ,fingiert') schon in der europäischen Debatte sehr unterschiedlich und oft unpräzise verwendet werden. Ein wichtiger Punkt betrifft die mangelnde Unterscheidung zwischen rhetorischer Inszenierung von vorgegebenem Material bzw. funktionaler Fiktionalität einerseits, und freier Erfindung bzw. autonomer Fiktionalität andererseits (hierzu Müller 2004: 282-283). So wechselt auch in der arabistischen Debatte die Verwendung der Termini von Autor zu Autor, und auch von Sprache zu Sprache (man denke z. B. an die spezifische Bedeutung des englischen ,fiction') und wird zudem mit den Oppositionen von wahr/unwahr, richtig/falsch, reell/imaginär, tatsächlich/nicht tatsächlich vermengt. Gerade Historiker neigen dazu, Fiktivität und Fiktionalität nicht ausreichend $\mathrm{zu}$ unterscheiden, so dass rhetorische und tendenziöse Geschichtsschreibung als ,Fiktion' betrachtet wird. ${ }^{21}$ Eine verwirrende Ambiguität ist schon in dem lateinischen Kernbegriff fictio angelegt, die hier allerdings nicht im Detail diskutiert werden kann (Korhonen 2006: 16); sie wird durch die Übertragung auf nichteuropäische Texttraditionen, die keinen äquivalenten Begriff haben, noch komplexer. Das Arabische kennt z. B. kadhib, das üblicherweise mit ,Lüge', ,Betrug', ,Unehrlichkeit' übersetzt wird; dies ist ein pejorativ bewertetes Konzept, das auch zur Abwertung von Trivialliteratur verwendet wird (s. o.). In manchen Kontexten, wie zum Beispiel bei der Kritik von Poesie, aber scheint kadhib auch so etwas wie literarische Kreativität bedeutet zu haben. ${ }^{22}$ Ferner gilt kadhib auch als Gegenteil von șidq, Wahrhaftigkeit, Ehrlichkeit' und bezieht sich somit nicht auf die Faktizität der Aussage, sondern auf die innere Haltung und eventuelle Täuschungsabsicht des Aussagenden.. ${ }^{23}$ Weitere Begriffe

\footnotetext{
Ähnlich Genette (1990: 762).

Vgl. Müller: „So ist die Diskussion einigermaßen verwirrend“ (2004: 283).

S. o. Anmerkung 8 und Leder (1998a: 51).

23 So vor allem in der Mystik, s. A. Knysh, Encyclopaedia of Islam, Second Edition, online, s.v. Șidk.
} 
sind $i k b t i r a \bar{a}^{c}$, was ebenfalls ,Kreativität, Erfindung ${ }^{6}$ in der Literatur heißen kann und sowohl im Zusammenhang mit Poesie (Heinrichs 1969) wie auch mit literarischer Prosa auftauchen kann (Leder 1998a: 48); ferner gibt es auch takhyill, ,Imagination' (Hoyland 2006: 17). Auf dem Feld der arabischen Terminologie und Konzeptualisierung sind noch viele Präzisierungen notwendig.

Im Folgenden möchte ich einige wichtige Linien des Diskussionsstrangs innerhalb der Arabistik zusammenfassend vorstellen und dann an einem Textbeispiel aus der arabischen Historiographie die Problematik illustrieren. ${ }^{24}$ Im Zentrum des Überblicks soll die umfangreiche und weiterhin sehr kontrovers geführte Debatte um ,Fakt und Fiktion' in der frühen arabischen Historiographie stehen, da sie wichtige Punkte berührt, die in der allgemeinen Fiktionalitätsfrage diskutiert werden.

\section{Fakt und Fiktion in der arabischen Geschichtsschreibung zum frühen Islam}

Die Diskussion um die Bewertung der frühislamischen Geschichtsschreibung zum frühen Islam füllt viele Seiten und hat sich inzwischen schon fast zu einem eigenen Forschungsgegenstand entwickelt, so dass hier nur ein skizzenhafter Überblick derjenigen Positionen gegeben werden kann, die für die hiesige Fragestellung relevant sind. ${ }^{25}$ Die Bedeutung dieser Debatte hängt damit zusammen, dass wir für die Frühzeit des Islams fast ausschließlich auf spätere narrative Quellen angewiesen sind, da uns dokumentarische Quellen kaum vorliegen und auch parallele, außerislamische Berichte rar sind (Donner 2011: 628; Donner 1998: 15). Jegliche Forschung zum frühen Islam muss sich somit letztlich mit der Hermeneutik arabischer Historiographie befassen.

Schon früh machte sich in der westlichen Forschung eine starke Skepsis breit, was die Historizität dieser historischen Berichte betrifft, wobei sich hier islamfeindliche, eurozentrische Vorurteile mit einer kritischen positivistischen Grundhaltung mischten. Im Zentrum dieses quellenkritischen Ansatzes stand die fragliche ,historische Zuverlässigkeit ${ }^{`}$ bzw. Historizität arabischer Historiographie. ${ }^{26}$ Eng damit verknüpft war die Diskussion über die Echtheit der prophetischen Überlieferung bzw. des hadìth, der sich ähnlicher Authentifizierungsstrategien wie die Historiographie bedient (ebenfalls isnād) und gleichfalls den Anspruch auf Faktualität er-

24 Die arabische Historiographie des Mittelalters ist auch im Sinne von Monika Fludernik narrativ und nicht argumentativ, da sie in ihrem Episodenformat anthropozentrische Erfahrung vermittelt. In ihrem modifizierten Narrativitätsmodell zählt sie nur den wissenschaftlichen historischen Diskurs, im Sinne der europäischen Moderne (2008: 73), zu den argumentativen Gattungen.

25 Die New Cambridge History of Islam widmet dieser Thematik sogar ein eigenes Kapitel: cf. Donner (2011).

26 Donner (1998: 8-12) ,source-critical approach“. 
hebt. ${ }^{27}$ Die Geschichtsschreibung und der hadìth wurden im Zuge dieser quellenkritischen Evaluierungen auf Anachronismen, Widersprüche und politischideologische Entstellungen geprüft und eine Liste ,unzuverlässiger ${ }^{6}$ Überlieferer, historischer Schulen und Autoren erstellt. Dennoch blieben die Kriterien letztlich subjektiv von dem Plausibilitätsempfinden des Forschers abhängig. So wurden die historischen Überlieferungen letztlich als Faktenberichte betrachtet, in denen ,richtige ${ }^{6}$ und ,falsche ${ }^{6}$ Traditionen voneinander zu scheiden seien, um in Rankescher Zielsetzung zu erforschen, „wie es eigentlich gewesen ist“ (Donner 1998: 9, 12).

Erst in den 70er Jahren kamen Ansätze aus der Literaturwissenschaft, Rhetorik und Bibelhermeneutik zum Einsatz. Albrecht Noth veröffentlichte 1973 seine bahnbrechende Studie Quellenkritische Studien zu Themen, Formen und Tendenzen frübislamischer Geschichtsüberlieferung, die in einer revidierten englischen Übersetzung 1994 publiziert wurde. Darin untersucht er erstmalig systematisch Themen, literarische Formen, Topoi und Schemata dieser Traditionen. Die Frage nach Fiktion und Fiktionalisierung stellt er zwar nicht direkt, aber er impliziert, dass topisches und literarisiertes Erzählen in diesen Texten zugleich ein Hinweis auf deren ,Unwahrheit' ist und setzt somit Literarisierung und ,Fiktion' gleich. ${ }^{28}$

Sein Buch erschien fast zeitgleich mit dem zentralen Buch Metahistory von Hayden White (1973) und reiht sich gewissermaßen in den ,narrative' und ,linguistic turn' der Kulturwissenschaften ein, ohne allerdings auf ihn Bezug zu nehmen.

Die frühen Arbeiten von Patricia Crone, welche das ,master narrative' der frühislamischen Geschichte dekonstruierten, seien hier nur kurz erwähnt, da deren Argumente nicht auf literaturwissenschaftlichen Ansätzen fußen. Die von ihren Publikationen ausgelöste Debatte ist aber insofern wichtig, da sie den wichtigen Impuls dazu gab, die Interpretation frühislamischer Geschichte erneut zu revidieren, und damit den ,skeptical approach'bzw. den Ansatz der Revisionisten begründete, der sich später auch dieser Begriffe bediente. ${ }^{29}$

Vor allem Stefan Leder widmete sich seit den späten 80ern eingehend der Frage nach den fiktionalen Elementen in der MHAL unter literaturwissenschaftlicher Perspektive, angeregt durch die Publikationen von Wolfgang Iser, wie Das Fiktive und das Imaginäre (1983), und seinen eigenen Forschungen zum Überlieferer al-Haytham b. 'Adī. In mehreren Publikationen untersucht er eingehend die literarischen Strategien historiographischer Texte (u. a. Leder 1990, Leder 1998a,

27 So vor allem die Arbeiten von Ignaz Goldziher und Joseph Schacht (Donner 1998: 12-16 „tradition-critical approach"). Sebastian Günther hat sich eingehend mit Fiktionalisierungsstrategien im badith beschäftigt und den Charakter dieser Überlieferungen als literarische Texte hervorgehoben (s. z. B. Günther 1998); seine Ergebnisse können hier allerdings nicht ausgeführt werden.

28 Noth (1973: 109-110); Donner (1998: 18-20); s. dazu auch die Kritik bei Meisami (2000: 16-18).

29 V. a. Crone/Cook (1977); zum ,skeptical approach's. den Überblick bei Donner (1998: 23-30). 
Leder 2005) und gab in diesen Zusammenhang auch einen Sammelband heraus (Leder 1998). In seinem programmatischen Aufsatz zum Thema ,Fiktion“ in der arabischen Literatur nennt er das Ziel, „to unmask the fictive narrative and thus prevent fiction from declaring itself a reality" (Leder 1998a: 46), eine Gefahr, die er als besonderes Problem der MHAL betrachtet (Leder 1998a: 34; cf. Leder 2005: 124). Seine Ausführungen sollen hier näher betrachtet werden:

So stellt er folgende Signale fest, die auf Fiktion hinweisen („detection of fiction and its use“, Leder 1998a: 46-57): 1. Die Verwendung von Humor, Ironie und anderen rhetorischen und stilistischen Mitteln, die eine Erzählung und ihren ,plot ${ }^{6}$ belehrend und zugleich unterhaltsam präsentieren (Leder 1998a: 4750, 56-59); 2. Die Existenz einer Rahmenerzählung, die den Akt des Erzählens selbst zum Gegenstand der Erzählung macht und damit auch eine orale Kommunikationssituation evoziert (Leder 1998a: 50); 3. Die Genre-Einordnung als Tierfabel, deren Inhalt aufgrund der Anthropomorphisierung von Tieren nur erfunden sein kann; Tierfabeln wurden dennoch ihrer Unterhaltsamkeit und Belehrung wegen auch in gelehrten Kreisen geschätzt (Leder 1998a: 51-52). In allen drei Fällen führt er Zeugnisse auf, die belegen, dass diese Erzählungen als fiktiv bzw. als fingierte Rede verstanden wurden.

Komplizierter gestaltet sich die Fragestellung im Falle der Historiographie, die ja den Anspruch auf Faktualität erhebt. Hierzu untersucht Stefan Leder in seinem Aufsatz exemplarisch die unterschiedlichen Versionen einer historischen Episode, die im Werk Ansāb al-ashrā $f$ des Historiographen Balādhūrī (gest. ca. 892) enthalten sind (Leder 1998a: 52-55). Die Gegenüberstellung verschiedener Fassungen bzw. akbbār ist, wie oben erwähnt, ein typisches Merkmal früher arabischer Historiographie. Stefan Leder weist nach, dass eine Version besonders stark fiktionalisiert ist („fictive elements [...] dominate“, „the more elaborate literary character [...] is evident to the reader") - und zwar diejenige, die Balādhūrì an das Ende seines Kapitels setzt. Diese Einordnung wertet er als „perception of decreasing factuality" (Leder 1998a: 55).

In seiner Schlussfolgerung stellt Stefan Leder allerdings fest, dass weder eine analytische Untersuchung des plots noch der narrativen Form einen zuverlässigen Hinweis auf den Status eines Textes gibt, sondern nur die intendierte Rezeption des Textes dies (zu leisten) vermag: „cultural contexts are of decisive importance“ (Leder 1998a: 59). Im Fall der arabischen Literatur kommt er zu dem Schluss, dass „narrative texts often oscillate between factual and fictive in pre-modern Arabic literature, because fiction has not won general acceptance as a mode of literary expression in its own right“ (Leder 1998a: 60; s. auch Leder 2005: 125-127).

Leders Argumentation ist unter anderem von Julie Meisami kritisiert worden (u. a. Meisami 2000: 16-18; s. o.). Sie betrachtet die rhetorische Inszenierung in der persischen und arabischen Historiographie als ein inhärentes Merkmal dieser Texte: 
Since history was, for them [gemeint sind die persischen Historiker und implizit auch die arabischen], less a dry record of events than an elucidation of the meaning of those events, historians employed such „literary devices“ - narrative structure, direct discourse, rhetorical embellishment, and so on - as would effectively convey that meaning. (Meisami 2000: 16)

Sie plädiert dafür, das mediävistische Konzept von ,ethical-rhetorical historiography' auf die arabische und persische Historiographie anzuwenden und betont, dass auch in den Fällen, in denen die frühe arabische Geschichtsschreibung relativ wenig ,embellished' (also literarisiert bzw. fiktionalisiert) erscheint, diese dennoch rhetorisch ist (Meisami 2000, 18; vgl. auch Beaumont 1996 und Shoshan 2004, X). Die rhetorische Dimension der arabischen Geschichtsschreibung und deren semantische Dichte sind in neuerer Zeit mehrfach untersucht worden, hier sei exemplarisch auf die Arbeiten von Tayyeb Hibri (2004 und 2010) und Boaz Shoshan (2004) verwiesen.

In einem neueren Übersichtsaufsatz widmete sich Robert Hoyland 2006 erneut der Frage nach der Rolle von Fiktion in der frühen arabischen Historiographie. Er schlug vor, scharfe Abgrenzungen zu vermeiden, und besser „history and fiction as lying on the same continuum" (Hoyland 2006: 18) zu betrachten. Dies empfiehlt sich umso mehr, als sich Historiographie in der Frühzeit noch nicht als eigenständige Disziplin etabliert hatte. In der Tat ist eine scharfe Trennung zwischen ,Literatur' (engl. ,fiction') und ,Geschichte' in der MHAL, wie sie auch in den Handbüchern reflektiert wird (Hoyland 2006: 18) eine Dichotomie, die von außen herangetragen wird, aber keine interne Differenzierung reflektiert.

Robert Hoyland unterstreicht noch einen weiteren Aspekt: Mittelalterliche arabische Autoren maßen die Echtheit einer khabar vorwiegend an der korrekten Überliefererkette, weniger an ihrem Inhalt (dies gilt ganz besonders für die Prophetentradition). Genau diese Strategie hat in der westlichen Forschung oft für Verwirrung gesorgt, führt sie doch dazu, dass (für den heutigen Leser!!) offensichtlich fiktive Inhalte als ,echt' eingestuft wurden (Hoyland 2006, 20-21).

Michael Cooperson stellte in einer Studie über das biographische Lexikon von al-Dhahabī (1274-1348) in diesem Zusammenhang eine sehr interessante These auf: In der islamischen Tradition sei „plausibility“ vor allem „in terms of probability" betrachtet worden (Cooperson 2005: 70). Sobald also eine Nachricht als glaubhaft eingestuft wurde, wurde deren Gültigkeit mit Hilfe der Überlieferungskette festgestellt, und so eine abschließende Entscheidung über die Faktizität ausgespart. Hier waltet die Erkenntnis, dass eine letztliche Gewissheit nicht zu erlangen sei, da die Umstände nicht mehr überprüfbar seien. Der normale Leser war dadurch „blessedly exent from the obligation of assessing it [its veracity]“ (Cooperson 2005: 77). Ohne Zweifel wäre es notwendig, die Vorstellungen von Plausibilität und Probabilität in der MHAL eingehender zu erforschen. 


\section{Faktuales Erzäblen? Die Gründung von Bagdad bei Tabarī}

Die Weltchronik bzw. die Annalen des TTabarī (839-923) gelten unbestritten als eines der Grundwerke der arabischen Historiographie. Der Autor war nicht nur ein angesehener Rechtsgelehrter, Koranexeget und Historiker - sondern besonders ein Experte darin, die meisten seiner Traditionen mit einem isnād, einer möglichst lückenlosen Überliefererkette, zuverlässig und anerkannt zu authentifizieren. Sein Werk gilt auch als ein typisches Beispiel für die schon erwähnte typische, ,molekulare' Struktur arabischer Historie, da er regelmäßig mehrere, teilweise widersprüchliche Nachrichten zum selben Ereignis gegenüberstellt, ohne diese zu harmonisieren und ein einheitliches Narrativ herzustellen. ${ }^{30}$

Für das Jahr 762 (145 Hidschra) berichtet er über ein epochales historisches Ereignis, nämlich über die Gründung der neuen abbasidischen Hauptstadt Bagdad durch den Kalifen al-Manșūr (reg. 754-775). Der Abschnitt umfasst in der englischen Übersetzung 13 Seiten und enthält mehrere widersprüchliche akbbār. ${ }^{31}$

Das Kapitel beginnt mit einer kurzen, realistischen kbabar, welche die wichtigsten Elemente nennt, die in den folgenden Versionen immer wieder erscheinen. ${ }^{32}$ Die Platzierung am Anfang legt nahe, dass Tabarī diese khabar für besonders glaubhaft hielt; das bemerkenswerte Fehlen einer Überliefererkette weist allerdings in eine andere Richtung und könnte somit ein ironischer Hinweis auf seine Skepsis sein. Diese erste khabar schildert kurz, wie sich al-Manșūr persönlich auf die Suche nach einem geeigneten Ort für die Stadtgründung begab, wobei er bei einem Ort südöstlich vom späteren Bagdad begann, über Bagdad zog und schließlich bis Mosul kam. Er kehrte dann nach Bagdad zurück, wo er eine kurze Rede hielt, in der er die Vorzüge des Ortes pries, worauf er persönlich den Grundriss der zukünftigen Stadt skizzierte und militärische Autoritäten einsetzte.

Auch wenn diese khabar im Stil realistisch-faktual gehalten ist, enthält sie einige Elemente, die vermutlich fiktiv sind. So ist es unwahrscheinlich, dass alManșūr, der sich zu diesem Zeitpunkt in der schwierigen Phase der Konsolidierung seiner Herrschaft befand, ein so großes Areal zeitraubend und ausführlich selbst absuchte. Die persönliche Suche des Herrschers ist zudem topisch, sie wird auch schon von Alexander dem Großen bei der Gründung von Alexandrien er-

30 Zum historischen Werk dieses Autors und seiner Arbeitsweise gibt es eine sehr umfangreiche Bibliographie; hier verweise ich stellvertretend auf die Arbeit von Boaz Shoshan (2004) und die dort genannte Literatur. Zur Chronographie im Islam s. den Überblick bei Robinson (2004: 74-79); zu Țabarī im Rahmen der arabischen Geschichtsschreibung seiner Zeit kurz Robinson (2004: 30-38).

31 Im Folgenden wird hier jeweils auf die englische Übersetzung des Werkes verwiesen; meiner deutschen Übersetzung lag allerdings auch der arabische Originaltext zugrunde: ațTabarī, Abū Ja'far Muhammad b.Ğarīr b.Yazìd, Ta'rī̄b ar-rusul wa-'l-mulūk wa-'l-bulafä', Ser. 1-3, Ed. M. J. de Goeje, Leiden 1879-1901; hier ser. III, 271-282.

32 Tabarī (915/1995) 237-238. Die khabar wird allerdings nicht als Paratext präsentiert, sondern als eine Tradition unter mehreren (fìmā dhukira - „man erzählt"). 
zählt. ${ }^{33}$ Das Motiv dient dem Zweck, der Stadtgründung eine besondere Bedeutung zu geben und unterstreicht auch die Handlungsfreiheit, Autorität und Fürsorge des Herrschers. Ebenso fiktiv ist höchstwahrscheinlich der Wortlaut der zitierten Rede des Kalifen, der die Vorzüge des Ortes hervorhebt. Fingierte Reden von historischen Persönlichkeiten sind ein häufiges literarisches Mittel zur Suggerierung von Faktualität in der arabischen Historiographie. ${ }^{34}$

Țabarī schließt nun eine khabar an, die im Gegensatz zur ersten mit einem isnād versehen und damit als faktual ,markiert' ist. Sie enthält allerdings fiktive Elemente, zumindest gemessen an unserem modernen Wahrheitsverständnis, und soll deshalb genauer betrachtet werden (Ṭabarī 915/1995: 238-240):

(isnād): Gemäß 'Umar b. Shahba - Muhammad b. Ma'rūf b. Suwayd - sein Vater - Sulaymān b. Muğālid: ${ }^{35}$

Die Leute in al-Kūfa ${ }^{36}$ wiegelten die Armee des Kalifen gegen ihn auf. So zog er in Richtung al-Ǧibāl auf der Suche nach einem Ort, um sich niederzulassen. Die Route ging damals über al-Madā'in. ${ }^{37}$ Wir verließen den Ort über al-Sabāt, aber einer unserer Kameraden blieb wegen einer Augenentzündung zurück. Als er dort blieb, um seine Augen heilen zu lassen, fragte ihn der Arzt: „Was sucht der Kalif?“ Er sagte: „Er sucht einen Ort, um sich niederzulassen." Da sagte [der Arzt]: „Wir haben in einem unserer Bücher gefunden, dass ein Mann namens Miqlāsa ${ }^{38}$ eine Stadt namens al-Zawrā’ zwischen dem Tigris und dem Șarāt bauen wird. Nachdem er ihren Grundriss gelegt hat und die Mauern gebaut hat, wird ihn die Nachricht über Probleme im Hiğăz erreichen. Dann wird er den Bau der Stadt unterbrechen und sich der Befriedung dieser Unruhen widmen, und sobald es ihm fast gelungen ist, wird er über Probleme in Bașra erfahren, die noch schwieriger für ihn als die ersten [im Hiğāz]. Aber er wird beide Probleme rasch lösen, und dann wird er den Bau [der Stadt] vollenden. Und er wird ein langes Leben haben, und die Herrschaft wird unter seinen Nachfolgern lange anhalten." Sulaymān fuhr fort: „Der Kalif war im Umfeld von al-Ğibāl und suchte einen Ort, um sich niederzulassen, als mein Kamerad zu mir kam und diese Geschichte erzählte; ich erzählte sie dann dem Kalifen. So ließ der Kalif den Mann holen, der ihm die Geschichte nochmals erzählte. Da kehrte der Kalif auf seinen Ausgangspunkt zurück und rief: ,Bei Gott, ich bin dieser! Denn ich wurde als Kind Miqlās genannt, aber danach wurde dieser Name nicht mehr für mich verwendet!'

33 Für eine detallierte Studie zur Bagdadlegende und den Parallelen vgl. Toral-Niehoff „Monks, Prophecies, Talismans and Divine Bliss - Legends about the Foundation of a Marvelous City“. Baghdad, Space of Knowledge. Hgg. Jens Scheiner und Isabel Toral-Niehoff (forthcoming) mit Verweis auf Plutarch, Vita Alexandri: 26, 1-10; vgl. auch O’Meara (2007).

34 Noth (1994: 87-96) „Speeches“.

35 Wie sich aus dem Text ergibt, war Sulaymān b. Muǧālid, ein ansonsten unbekannter Mann, in der Entourage des Kalifen, als er sich auf die Suche begab. Er ist der Erzähler und Augenzeuge. Die anderen Namen sind bekannte Überlieferer.

36 Stadt im südlichen Irak, bekannt für die schiitischen Sympathien seiner Bewohner. Hier fanden mehrere Revolten statt, die die Herrschaft der Abbasiden bedrohten.

37 Al-Madā'in (ehemaliges Ktesiphon-Seleukeia) und Kūfa sind bekannte Städte im südlichen Irak - aber auch alle anderen hier erscheinenden Ortsnamen sind identifizierbar. Für Details sei auf die kommentierte engl. Übersetzung verwiesen.

38 Der Name Miqlāṣ ist ungewöhnlich und für al-Manșūr außerhalb dieser Anekdote (die allerdings in Varianten immer wieder zitiert wird) nicht bezeugt. 
Diese khabar enthält eine typische Prophezeiung ex eventu, welche die besondere Bedeutung des Gründungsaktes und damit der Stadt selbst unterstreichen soll. Das Motiv des weisen Mannes, der in seinen Büchern eine Prophezeiung findet, die das ruhmreiche Schicksal der Stadt ankündigt, ist ein topisches Element, das sich regelmäßig in islamischen Stadtgründungslegenden findet. Vermutlich geht es auf ältere lokale Modelle zurück. ${ }^{39}$ Aus moderner Sicht ist eine solche Prophezeiung unwahr, da sie unseren Vorstellungen von Rationalität nicht entspricht.

Analysieren wir den fiktionalen bzw. faktualen Status der khabar, fällt es allerdings schwer, einzuschätzen, wie Tabarī die khabar verstand, ob er sich also des fiktiven Charakters der khabar bewusst war. Der Stil ist kaum literarisiert, realistisch und szenisch, Humor und Ironie sind nicht enthalten. Zahlreiche Details, wie z. B. die akkurate Nennung von existierenden Ortsnamen und historischen Personen, evozieren Realitätsnähe. Der Erzähler ist ein Augenzeuge, interveniert aber kaum im Geschehen. Auch der paratextuelle Kontext suggeriert die Interpretation als faktualen Bericht: Er ist in einen Rahmen eingebettet, der kein ,Als-ob“ suggeriert. In der historischen Chronik des seriösen Gelehrten Țabarī wird er von einer Überlieferungskette begleitet, die den Ansprüchen an Authentizität genügt. Innerhalb des Gesamtabschnittes zur Gründung Bagdads befindet sich diese khabar zudem an zweiter Stelle - gemäß der Regel, dass die Reihenfolge der akbbār eine „decreasing perception of factuality“ (Leder 1998a: 55) bedeutet, hieße das, dass Țabarī die Nachricht für plausibel hielt, jedenfalls für glaubwürdiger als die darauf folgenden. Schließlich hält sich Țabarī mit paratextuellen Hinweisen, die auf seine Haltung hinweisen würden, bemerkenswert zurück. So verweist er nicht auf eine wundersame Erfüllung der Prophezeiung, und somit auf deren Wahrheitsgehalt, sondern überlässt das Urteil darüber dem Leser.

Die nächsten akbbār des Kapitels seien hier nur kurz zusammengefasst, um den textuellen Kontext einzuordnen (Ṭabarī 915/1995: 240-251). Einige gehen ebenfalls auf den Augenzeugen Sulaymān b. Muǧālid zurück (allerdings mit anderem isnād). Sie schildern im Detail die persönlichen Bemühungen des Kalifen, den rechten Ort zu finden, zitieren jeweils die Lobrede des Kalifen in mehreren längeren, recht unterschiedlichen Varianten, und beschreiben schließlich auch dessen genaue Anweisungen für die Planung und den Grundriss der neuen Stadt. Die Prophezeiung mit dem geheimnisvollen Namen Miqlāṣ erscheint an anderer Stelle erneut, leicht abgewandelt, und nun unter Berufung auf einen anderen Gewährsmann (Ṭabarī 915/1995, 244). In diesem Fall fragt der Kalif direkt einen Mönch nach einer Prophezeiung, setzt also die Existenz einer solchen voraus; diese khabar dürfte also sekundär zur ersten Geschichte über Miqlāṣ entstanden sein.

Betrachten wir den Abschnitt in einem weiteren kulturellen Kontext, lässt sich auch kaum entscheiden, ob und inwieweit Țabarī den Bericht als glaubhaft bzw. historisch betrachtete. Der Glaube an Prophezeiungen und ähnliche Wunder war weit

39 O’Meara (2007) und Toral-Niehoff (forthcoming), s. o. Anm. 32. 
verbreitet, und Teil des damaligen Weltwissens. Die Ähnlichkeit der MiqlāṣGeschichte mit der islamischen Bahïra-Legende (wonach der christliche Mönch Bahìra dem Propheten Muhammad die ruhmreiche Zukunft des Islam voraussagte $)^{40}$ gab dieser Erzählung in den Augen frommer Muslime zusätzliche Plausibilität.

Das gesamte Kapitel verdeutlicht, dass es nicht die Zielsetzung von Ṭabarī war, historisch zuverlässige ,Fakten'-Berichte zur Gründung Bagdads im Sinne positivistischer Geschichtsschreibung (,wie es wirklich gewesen ist“) zu präsentieren. Die Frage ,Fakt oder Fiktion' stand also gar nicht im Zentrum seiner Darstellung und blieb gewissermaßen offen. Vielmehr standen zwei Themen im Vordergrund: Die zentrale Autorität und Fürsorge des Kalifen, der sich um jedes Detail der Stadtplanung persönlich kümmerte, und die besondere, schicksalhafte Bedeutung der Stadt Bagdad. TTabarī schrieb in einer politisch bewegten und krisenhaften Epoche, die durch einen massiven Machtverlust des Kalifats zugunsten anderer politischer Kräfte gekennzeichnet war. In diese Zeit fällt die Verklärung der ersten Abbasidenzeit und des frühen Bagdad zu einem ,goldenen' Zeitalter, ein Bild, das zu einer romantisierten Projektionsfläche für spätere Generationen wurde. Dies ist der historische Kontext, in den dieser Abschnitt einzuordnen und $\mathrm{zu}$ interpretieren ist. ${ }^{41}$

\section{Schlussbemerkungen}

Wie dieses Beispiel und die Debatte zeigen, bedient sich die frühislamische Historiographie zwar einer realistisch-faktualen Erzählweise, entzieht sich aber einer klaren Kategorisierung als faktuales oder fiktionales Erzählen. Zumindest gab es keine explizite Entblößung des Fiktionscharakters, die helfen würde, diese zu erkennen. Die Verwendung fiktiver Elemente, die oft als Kriterium für den Fiktionscharakter mancher Passagen der MHAL-Prosa gelten, kann nicht als Hinweis zugrunde gelegt werden, so lange nicht deutlich wird, ob sich der Autor dieser Fiktivität bewusst war. Eine Konzeptualisierung von Fiktion fand in der MHAL auch kaum statt, vergleichbare Vorstellungen in der hoch entwickelten arabischen Literaturkritik und Rhetorik des Mittelalters sollten jedoch noch vertieft untersucht werden.

Grundsätzlich lässt sich festhalten, dass die MHAL sehr viele Parallelen zur mittelalterlichen europäischen Literatur und Historiographie aufweist und somit auch ähnliche Probleme bereitet, wenn Fiktionalitätsmodelle angewendet werden, die an der europäischen Moderne orientiert sind. So fehlt in beiden Fällen ein ausdifferenziertes, autonomes System Literatur, das einen Rahmen setzen könnte, wann eine Aussage als fiktional zu gelten hat; eine explizite Ausstellung des Fiktionscharakters fällt also notwendigerweise aus.

40 Abel, A., Bahīiā , in Encyclopaedia of Islam, $2^{\text {nd }}$ edition, online.

41 Zur Konstruktion der, Goldenen Zeit' in Bagdad vgl. Cooperson (2006) und Hibri (2004: 17-58). 
Die Mediävistik hat deshalb Modifikationen und Präzisierungen entwickelt, die auch für die Arabistik fruchtbar gemacht werden könnten. Jan D. Müller plädiert z. B. für ein ,weiches' Fiktionalitätsmodell, das es erlaubt, auch ohne explizite ,Ausstellung' des Fiktionscharakters einen Text als fiktional zu betrachten, falls „die (jederzeit mögliche) Aufdeckung des Fiktionscharakters den Geltungsund Wahrheitsanspruch der Aussage nicht nur nicht zerstört, sondern überhaupt nicht tangiert“ (Müller 2004: 285). Dies würde es erlauben, Texte des adab als fiktional zu betrachten, auch wenn der Stil Faktualität evoziert, auf historische Begebenheiten referiert und sich nicht offen als Fiktion erklärt. Auch der Begriff ,Vorzeitkunde' (Müller 2004: 286) könnte sinnvoll sein, um viele Arten des ,Erzählens über die Vergangenheit' in der MHAL zu fassen, ohne in eine Dichotomie Geschichte vs. Literatur zu verfallen.

Hier sind nur einige Beispiele aus dem breiten Repertoire von Konzepten und Theorien aus der Mediävistik genannt, die in der Arabistik Anwendung finden könnten. Umgekehrt dürfte die Mediävistik und Komparatistik von der Arabistik profitieren, da sie so außereuropäische Modi der Fiktion, die anderen Traditionen und theoretischen Konzepten unterliegen, in ihre Überlegungen mit einbezieht. Denn es zeigt sich am Beispiel der arabischen Literatur des Mittelalters deutlich, dass „Fingieren kulturspezifisch sehr vielfältige Bedeutungen und Funktionen" hat (Müller 2004: 283).

\section{Literatur}

Abel, A. „Bahịīā". Encyclopaedia of Islam. $2^{\text {nd }}$ edition. Online.

Beaumont, Daniel (1996) „Hard-Boiled: Narrative Discourse in Early Muslim Traditions". Studia Islamica 83: 5-31.

Bonebakker, Seger A. (1990) „Adab and the Concept of Belles-Lettres“. Abbasid Belles-Lettres. The Cambridge History of Arabic Literature. Hgg. Julia Ashtiany et alii. Cambridge: Cambridge Univ. Press. 16-30.

- (1992) „Some Medieval Views of Fantastic Stories“. Quaderni di Studi Arabi 10: 21-43.

Bürgel, Johann Christoph (1990) „Die persische Epik“. Neues Handbuch der Literaturwissenschaft. Orientalisches Mittelalter. Bd. 5. Hg. Wolfhart Heinrichs. Wiesbaden: Akademie Verlag. 301-18.

Caskel, Werner (1930) „Aijam al-`Arab. Studien zur altarabischen Epik“. Islamica 3.5: 1-99.

Cooperson, Michael (2005) „Probability, Plausibility and ,Spiritual Communication" in Classical Arabic Biography". On Fiction and Adab in Medieval Arabic Literature. Hg. Philip Kennedy. Wiesbaden: Harrassowitz. 69-84.

- (2006) „Baghdad in Rhetoric and Narrative“. Muqarnas 13: 99-113.

Crone, Patrica, und Michael Cook (1977) Hagarism: The Making of the Islamic World. Cambridge: Cambridge Univ. Press. 
Donner, Fred M. (1998) Narratives of Islamic Origins: The Beginnings of Islamic Historical Writing. Princeton: Darwin Press.

- (2011) „Modern Approaches to Early Islamic History“. The Nerw Cambridge History of Islam. Hg. Chase F. Robinson. 625-44.

Drory, Rina (1994) „Three Attempts to Legitimize Fiction in Classical Arabic Literature“. Jerusalem Studies in Arabic and Islam 18: 289-307.

Fähndrich, Hartmut (1990) „Der Begriff «adab» und sein literarischer Niederschlag“. Neues Handbuch der Literaturwissenschaft. Orientalisches Mittelalter. Bd. 5. Hg. Wolfhart Heinrichs. Wiesbaden: Akademie Verlag. 326-45.

Fludernik, Monika (2008) Erzäbltheorie. Eine Einfübrung [2006]. Darmstadt: Wissenschaftliche Buchgesellschaft.

Genette, Gérard, Nitsa Ben-Ari und Brian McHale (1990) „Fictional Narrative, Factual Narrative“. Poetics Today 11.4: 755-74.

Ghazi, Mohammed Ferid (1957) „La litterature d'imagination en arabe du IIe/VIIIe au Ve/XIe siècles“. Arabica 4.2: 164-68.

Grunebaum, Gustave von (1947) Medieval Islam. Chicago: University of Chicago Press.

Günther, Sebastian (1998) „Fictional and Imagination within an Authoritative Framework: Towards a New Understanding of Hadith“. Story-telling in the Framework of Non-Fictional Arabic Literature. Hg. Stefan Leder. Wiesbaden: Harrassowitz. 433-71.

Haug, Walter (2003) „Die Entdeckung der Fiktionalität“. Die Wabrheit der Fiktion: Studien zur weltlichen und geistlichen Literatur des Mittelalters und der frühen Neuzeit. Hg. Walter Haug. Tübingen: Max Niemeyer. 128-44.

Heath, Peter (1990) „Arabische Volksliteratur im Mittelalter“. Neues Handbuch der Literaturwissenschaft. Orientalisches Mittelalter. Bd. 5. Hg. Wolfhart Heinrichs. Wiesbaden: Akademie Verlag. 423-39.

Heinrichs, Wolfhart (1969) Arabische Dichtung und Griechische Poetik: Häżim alQartağannīs Grundlegung der Poetik mit Hilfe Aristotelischer Begriffe. Beiruter Texte und Studien. Hg. vom Orient Institut der Deutschen Morgenländischen Gesellschaft. Beirut: Franz Steiner Verlag.

Hibri, Tayyeb (2004) Reinterpreting Islamic Historiography: Hārūn al-Rashīd and the Narrative of the 'Abbasid Caliphate. Cambridge: Cambridge Univ. Press.

- (2010) Parable and Politics in Early Islamic History. New York: Columbia Univ. Press.

Hoyland, Robert C. (2006) „History, Fiction and Authorship in the First Centuries of Islam“. Writing and Representation in Early Islam: Muslim Horizons. Hg. Julia Bray. London: Routledge. 16-46.

Ibn al-Nadīm, Muhammad Abū 1-Faradj (1871/2) Kitāb al-Fibrist. Hg. Gustav Flügel. Leipzig: Vogel.

Iser, Wolfgang (1983) Das Fiktive und das Imaginäre: Perspektiven einer literarischen Anthropologie. München: Suhrkamp. 
Kennedy, Philip F. (2005) Hg. On Fiction and Adab in Medieval Arabic Literature. Wiesbaden: Harrassowitz.

- (2005a) „Preface“. On Fiction and Adab in Medieval Arabic Literature. Hg. Philip F. Kennedy. Wiesbaden: Harrassowitz. xi-xxii.

Knysh, A. „Șidk“. Encyclopaedia of Islam. $2^{\text {nd }}$ edition. Online.

Korhonen, Kuisma (2006) Hg. Tropes for the Past: Hayden White and the History/Literature Debate. Internationale Forschungen zur Allgemeinen und Vergleichenden Literaturwissenschaft 96. Amsterdam/New York: Rodopi.

Leder, Stefan (1990) „Features of the Novel in Early Historiography: the Downfall of Xālid al-Qasrī“. Oriens 32: 72-96.

- (1992) „The Literary Use of the Khabar: A Basic Form of Historical Writing“. The Byzantine and Early Islamic Near East I: Problems in the Literary Source Material. Hgg. Averil Cameron und Lawrence Conrad. Princeton: Darwin Press. 277-315.

- (1998) Hg. Story-Telling in the Framerwork of Non-Fictional Arabic Literature. Wiesbaden: Harrassowitz.

- (1998a) "Conventions of Fictional Narration in Learned Literature“. Storytelling in the Framework of Non-Fictional Arabic Literature. Hg. Stefan Leder. Wiesbaden: Harrassowitz. 34-60.

- (2005) „The Use of Composite Form in the Making of the Islamic Historical Tradition". On Fiction and Adab in Medieval Arabic Literature. Hg. Philip F. Kennedy. Wiesbaden: Harrassowitz. 125-48.

Leder, Stefan, und Hilary Kilpatrick (1992) „Classical Arabic Prose Literature. A Researcher's Sketch map“. Journal of Arabic Literature 23.1: 2-26.

Meisami, Julie Scott (2000) „History as Literature“. Iranian Studies 33.1.2: 15-30.

- (2005) „Mas' ūdī and the Reign of al-Aminn: Narrative and Meaning in Medieval Muslim Historiography“. On Fiction and Adab in Medieval Arabic Literature. Hg. Philip F. Kennedy. Wiesbaden: Harrassowitz. 149-76.

Meisami, Julie Scott, und Paul Starkey (1998) Encyclopaedia of Arabic Literature. 2 Bde. London/New York: Routledge.

Müller, Jan-Dirk (2004) „Literarische und andere Spiele: Zum Fiktionalitätsprinzip in vormoderner Literatur". Poetica: Zeitschrift für Sprach- und Literaturwissenschaft 36: 281-312.

Noth, Albrecht (1973) Quellenkritische Studien zu Themen, Formen und Tendenzen frühislamischer Geschichtsüberlieferung. Bonn: Selbstverlag des Orientalischen Seminars der Universität. Überarbeitete englische Fassung (in Kooperation mit Laurence Conrad, 1994): The Early Arabic Historical Tradition. Princeton: Princeton Univ. Press.

O’Meara, Simon (2007) „The Foundation Legend of Fez and Other Islamic Cities in the Light of the Life of the Prophet". Cities in the Pre-Modern Islamic World. Hgg. Amira Bennison et alii. London/New York: Routledge. 27-41.

Rosenthal, Franz (1968) A History of Muslim Historiography. Brill: Leiden. 
Robinson, Chase F. (2004) Islamic Historiography [2003]. Cambridge: Cambridge Univ. Press.

Schock, Fleming (2012) Hg. Polyhistorismus und Buntschriftstellerei: Populäre Wissensformen und Wissenskultur in der Frühen Neuzeit. Berlin/Boston: De Gruyter.

Shoshan, Boaz (2004) Poetics of Islamic Historiography: Deconstructing Tabarì's History. Islamic History and Civilization 53. Studies and Texts. Hgg. Wadad Kadi und Rotraud Wieland. Leiden/New York: Brill.

Toral-Niehoff (forthcoming) „Monks, Prophecies, Talismans and Divine Bliss Legends about the Foundation of a Marvelous City“. Baghdad, Space of Knowledge. Hgg. Jens Scheiner und Isabel Toral-Niehoff.

Ṭabarī, Abū Ğa far Muhammad (915/1995) The History of al-Tabarī, vol. XXVIII: 'Abbāsid Authority Affirmed. Übers. Jane D. McAuliffe. New York: State Univ. New York Press.

White, Hayden (1973) Metahistory: The Historical Imagination in Nineteenth-Century Europe. Baltimore/London: John Hopkins Univ. Press.

\section{Glossar}

khabar, pl. akbbār: Kurze Nachricht oder Bericht, grundlegende narrative Einheit in der frühen arabischen Prosa.

isnād: Überliefererkette, die als Authentifizierung einer khabar dient und möglichst lückenlos bis zum ersten Tradenten führt, der idealiter ein Augenzeuge oder zumindest ein Zeitgenosse des Ereignisses ist.

$a d a b$ : vielgestaltige Textgruppe, am ehesten entspricht diese Gruppe der mittelalterlichen und neuzeitlichen ,Buntschriftstellerei', oft auch für gesamte literarische Prosa in klassischem Arabisch verwendet. Impliziert ist auch ein Bildungsideal und die Vorstellung von Kultiviertheit.

haditth: Tradition über das Leben und die Aussprüche des Propheten, wurde schließlich zu einer wichtigen Rechtsquelle im Islam. Hier ist die Authentifizierung durch einen korrekten isnād besonders wichtig. 
